\title{
SOME PROPERTIES OF ORDINARY SENSE SLICE 1-LINKS: SOME ANSWERS TO PROBLEM (26) OF FOX
}

\author{
EIJI OGASA
}

(Communicated by Ronald A. Fintushel)

\begin{abstract}
We prove that, for any ordinary sense slice 1-link $L$, we can define the Arf invariant, and $\operatorname{Arf}(L)=0$. We prove that, for any $m$-component 1-link $L_{1}$, there exists a $3 m$-component ordinary sense slice 1-link $L_{2}$ of which $L_{1}$ is a sublink.
\end{abstract}

\section{INTRODUCTION AND MAIN RESULTS}

In [3] Fox submitted the following problem about 1-links. Note that "slice link" in the following problem is now called "ordinary sense slice link," and "slice link in the strong sense" is now called "slice link" by knot theorists.

Problem 26 of [3]. Find a necessary condition for $L$ to be a slice link; a slice link in the strong sense.

Our purpose is to give some answers to the first part of this problem. The latter half is not discussed here. The latter half seems discussed much more often than the first half. See e.g. [2], [5] and [14].

We review the definitions of ordinary sense slice links and of slice links, which we now use.

We suppose $m$-component 1-links are oriented and ordered.

Let $L=\left(K_{1}, \ldots, K_{m}\right)$ be a $m$-component 1-link in $S^{3}=\partial B^{4} . L$ is called a slice 1-link, which is "a slice link in the strong sense" in Fox's terminology, if there exist 2-discs $D_{i}^{2}(i=1, \ldots, m)$ in $B^{4}$ such that $D_{i}^{2} \cap \partial B^{4}=\partial D_{i}^{2}, D_{i}^{2} \cap D_{j}^{2}=\varnothing(i \neq j)$, and $\left(\partial D_{1}^{2}, \ldots, \partial D_{m}^{2}\right)$ in $\partial B^{4}$ defines $L$.

Take a 1-link $L$ in $S^{3}$. Take $S^{4}$, and regard $S^{4}$ as $\left(\mathbb{R}^{3} \times \mathbb{R}\right) \cup\{\infty\}$. Regard the 3 -sphere $S^{3}$ as $\mathbb{R}^{3} \cup\{\infty\}$ in $S^{4}$. L is called an ordinary sense slice 1-link, which is "a slice link" in Fox's terminology, if there exists an embedding $f: S^{2} \hookrightarrow \mathbb{R}^{3} \times \mathbb{R}$ such that $f$ is transverse to $\mathbb{R}^{3} \times\{0\}$ and $f\left(S^{2}\right) \cap\left(\mathbb{R}^{3} \times\{0\}\right)$ in $\mathbb{R}^{3} \times\{0\}$ defines $L$. Suppose $f$ defines a 2 -knot $X$. Then $L$ is called a cross-section of the 2-knot $X$.

From now on we use the terms in their currently accepted senses.

Ordinary sense slice 1-links have the following properties.

Received by the editors April 10, 1996 and, in revised for, December 27, 1996.

1991 Mathematics Subject Classification. Primary 57M25, 57Q45.

Key words and phrases. Ordinary sense slice 1-links, Arf invariants, $n$-dimensional knots and links, Suzuki-Terasaka diagrams, realizable 4-tuple of links.

This research was partially supported by Research Fellowships of the Promotion of Science for Young Scientists.

(C)1998 American Mathematical Society 
Theorem 1.1. Let $L$ be an ordinary sense slice 1-link. Then the following hold.

(1) $L$ is a proper link.

(2) $\operatorname{Arf}(L)=0$.

Note. Although our proof proves (1) and (2) simultaneously, once (1) is known (2) follows easily from the known result that a proper link which is an ordinary sense slice link has trivial Arf invariant. See e.g. [4], [9], [16] and [19].

Theorem 1.2. For any $m$-component 1 -link $L$, there exists a $3 m$-component ordinary sense slice 1-link $L^{\prime}$ of which $L$ is a sublink.

Note. When $L$ is not slice and $m=1$, it is obvious that ' $3 m$ ' is best possible. When $m \geqq 2, ' 3 m$ ' is not best possible even if no components of $L$ are slice knots. See the example for Note 3.3 and Figure IV in $\S 3$.

Theorem 1.2 follows from Theorem 1.3 obviously.

Theorem 1.3. For any $m$-component 1-link $L=\left(K_{1}, \ldots, K_{m}\right)$, there exist an embedding $f: S_{1}^{2} \amalg \ldots \amalg S_{m}^{2} \hookrightarrow \mathbb{R}^{3} \times \mathbb{R}$ and a $3 m$-component 1-link $L^{\prime}$ with the following properties.

(1) $f$ is transverse to $\mathbb{R}^{3} \times\{0\}$, and $f\left(S_{1}^{2} \amalg \ldots \amalg S_{m}^{2}\right) \cap\left(\mathbb{R}^{3} \times\{0\}\right)$ in $\mathbb{R}^{3} \times\{0\}$ defines $L^{\prime}$.

(2) $L$ is a sublink of $L^{\prime}$.

(3) $K_{i} \subset f\left(S_{i}^{2}\right)(i=1, \ldots, m)$.

This paper is organized as follows. In $\S 2$ we prove Theorem 1.1 by using a result of the author's [17]. In $\S 3$ we review Suzuki-Terasaka diagrams of 1-links and the fact that any 1-link has a Suzuki-Terasaka diagram (Theorem 3.1). We use this diagram to prove Theorem 1.3 and Theorem 3.2.

\section{The Proof of Theorem 1.1}

In order to prove Theorem 1.1, we review a result of the author in [17].

Definition. $T=\left(L_{1}, L_{2}, X_{1}, X_{2}\right)$ is called a 4 -tuple of links if the following conditions (1), (2) and (3) hold.

(1) $L_{i}=\left(K_{i 1}, \ldots, K_{i m_{i}}\right)$ is an oriented ordered $m_{i}$-component 1-dimensional link $(i=1,2)$.

(2) $m_{1}=m_{2}$.

(3) $X_{i}$ is an oriented 3-knot.

Definition. A 4-tuple of links $\left(L_{1}, L_{2}, X_{1}, X_{2}\right)$ is said to be realizable if there exists a smooth transverse immersion $f: S_{1}^{3} \amalg S_{2}^{3} \rightarrow S^{5}$ satisfying the following conditions (1) and (2).

(1) $f \mid S_{i}^{3}$ is a smooth embedding and defines the 3 -knot $X_{i}(i=1,2)$ in $S^{5}$.

(2) For $C=f\left(S_{1}^{3}\right) \cap f\left(S_{2}^{3}\right)$, the inverse image $f^{-1}(C)$ in $S_{i}^{3}$ defines the 1-link $L_{i}(i=1,2)$. Here, the orientation of $C$ is induced naturally from the preferred orientations of $S_{1}^{3}, S_{2}^{3}$, and $S^{5}$, and an arbitrary order is given to the components of $C$.

The following theorem characterizes the realizable 4-tuples of links.

Theorem 2.1. A 4-tuple of links $T=\left(L_{1}, L_{2}, X_{1}, X_{2}\right)$ is realizable if and only if $T$ satisfies one of the following conditions $i)$ and ii). 
i) Both $L_{1}$ and $L_{2}$ are proper links, and

$$
\operatorname{Arf}\left(L_{1}\right)=\operatorname{Arf}\left(L_{2}\right) .
$$

ii) Neither $L_{1}$ nor $L_{2}$ is proper, and

$$
\operatorname{lk}\left(K_{1 j}, L_{1}-K_{1 j}\right) \equiv \operatorname{lk}\left(K_{2 j}, L_{2}-K_{2 j}\right) \quad \bmod 2 \text { for all } j .
$$

Note. In [18], the author discussed a high-dimensional version of Theorem 2.1.

Proof of Theorem 1.1. Take $f: S^{2} \hookrightarrow \mathbb{R}^{3} \times \mathbb{R}$ such that $L$ is a cross-section of the 2-knot defined by $f$.

Regard $\mathbb{R}^{3} \times \mathbb{R}$ as $\mathbb{R}^{3} \times \mathbb{R} \times\{0\} \subset \mathbb{R}^{3} \times \mathbb{R} \times \mathbb{R}$. Make $S^{5}$ from $\mathbb{R}^{3} \times \mathbb{R} \times \mathbb{R}$ by the one-point compactification. Here, the 4 -sphere $S^{4}$ is $\left(\mathbb{R}^{3} \times \mathbb{R} \times\{0\} \cup\{\infty\}\right) \subset$ $S^{5}=\left(\mathbb{R}^{3} \times \mathbb{R} \times \mathbb{R} \cup\{\infty\}\right)$. There exists a 3 -knot $X_{1}$ in $\mathbb{R}^{3} \times \mathbb{R} \times \mathbb{R}$ such that $X_{1}$ $\cap \mathbb{R}^{3} \times \mathbb{R} \times\{0\}$ coincides with $f\left(S^{2}\right)$, because all 2-knots are slice by a theorem of Kervaire in [10]. $\left(\mathbb{R}^{3} \times\{0\} \times\{0\}\right) \cup\{\infty\}$ in $S^{5}$ is called a 3 - knot $X_{2}$. An immersion $g: S_{1}^{3} \amalg S_{2}^{3} \rightarrow S^{5}$ such that $g\left(S_{i}^{3}\right)$ coincides with the above $X_{i}(i=1,2)$ realizes a pair of 1-links $\left(X_{1} \cap X_{2}\right.$ in $X_{1}, X_{1} \cap X_{2}$ in $\left.X_{2}\right)$. Here, it is obvious that $X_{1} \cap X_{2}$ in $X_{1}$ is the trivial 1-link and $X_{1} \cap X_{2}$ in $X_{2}$ is $L$. Therefore, by Theorem 2.1,L is a proper link and $\operatorname{Arf}(L)=0$.

\section{The Proof of Theorem 1.3}

We first review the following canonical diagrams of 1-links.

Take an $m$-component 1-link $L$. The set $X$ in $\mathbb{R}^{3}=\{(x, y, z)\}\left(\subset S^{3}\right)$ defining $L$ is called a Suzuki-Terasaka (canonical) diagram of $L$ if $X$ is made as follows. Let $Y_{i}$ $(i=1, \ldots, m)$ be the boundary of $\{(x, y, z) \mid i \leqq x \leqq(i+0.9), 0 \leqq y \leqq 1, z=0\}$. Let $P_{i j}\left(j=1, \ldots, \mu_{i}\right)$ be the boundary of $\left\{(x, y, z) \mid x=\frac{j}{\mu_{i}+1}+i, 0.9 \leqq y \leqq 1.1,-0.1 \leqq\right.$ $z \leqq 0.1\}$. (The orientation of $P_{i j}$ is given appropriately; they are not all the same, in general.) Let $A_{i j}=\left\{(x, y, z) \mid \frac{2 j-1}{2 \nu_{i}+1}+i \leqq x \leqq \frac{2 j}{2 \nu_{i}+1}+i, y=0, z=0\right\}\left(j=1, \ldots, \nu_{i}\right)$. Let $\sum_{i}^{m} \mu_{i}=\sum_{i}^{m} \nu_{i}$; put $\lambda$ equal to this number. Take bands $B_{l}(l=1, \ldots, \lambda)$ and make a band-sum of $Y_{i}$ and $P_{i j}$ by connecting $P_{i j}$ and $A_{i^{\prime} j^{\prime}}$ by $B_{l}$. (Of course $(i, j)$ does not necessarily coincide with $\left(i^{\prime}, j^{\prime}\right)$, and the set of $(i, j)$ coincides with the set of $\left(i^{\prime}, j^{\prime}\right)$.) Then the band-sum is $X$.

Theorem 3.1. Any 1-link has a Suzuki-Terasaka canonical diagram.

The proof of Theorem 3.1 is elementary.

The usefulness of the above canonical diagram was first pointed out by Prof. Shin'ichi Suzuki and Prof. Hidetaka Terasaka. Suzuki-Terasaka canonical diagrams are used, for example, in [15], [23] and [25].

In Figure I we give an example of the canonical diagram.

Proof of Theorem 1.3. Take a Suzuki-Terasaka canonical diagram of the 1-link $L=\left(K_{1}, \ldots, K_{m}\right)$ in $\mathbb{R}^{3}=\{(x, y, z)\} \quad\left(\subset S^{3}\right)$. (See Figure II(1).) Take sets $P_{i}$ to be $\{(x, y, z) \mid i \leqq x \leqq(i+0.9), 1.05 \leqq y \leqq 2, z=0\}$. Here, we can take $P_{i}$ not to intersect with all the bands in the Suzuki-Terasaka canonical diagram. Take sets $S_{i}$ to be $\{(x, y, z) \mid i \leqq x \leqq(i+0.9),-1 \leqq y \leqq 1, z=0\}$. Note that $K_{i} \cap S_{i} \neq$ $\phi$. (See Figure II(2).) The arc in $K_{i}$ whose boundary is the points $\{(i, 0,0)\}$ and $\{(i+0.9,0,0)\}$ and which does not include the point $\{(i, 1,0)\}$ is called $l_{i}$. Carry 


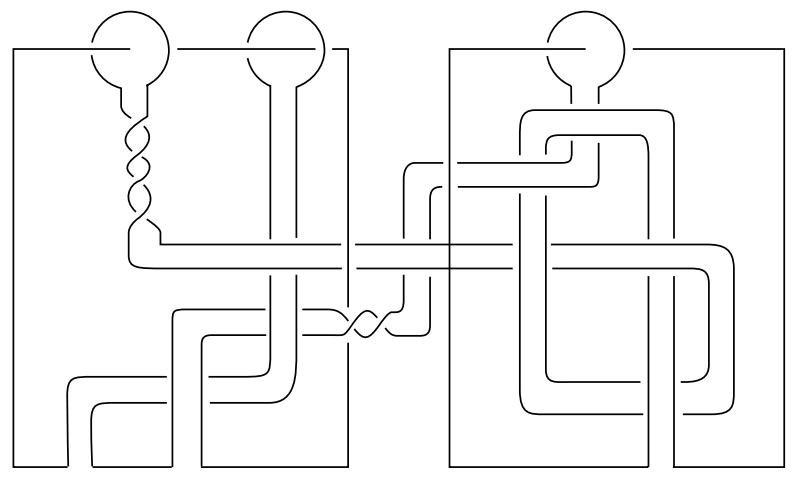

Figure I

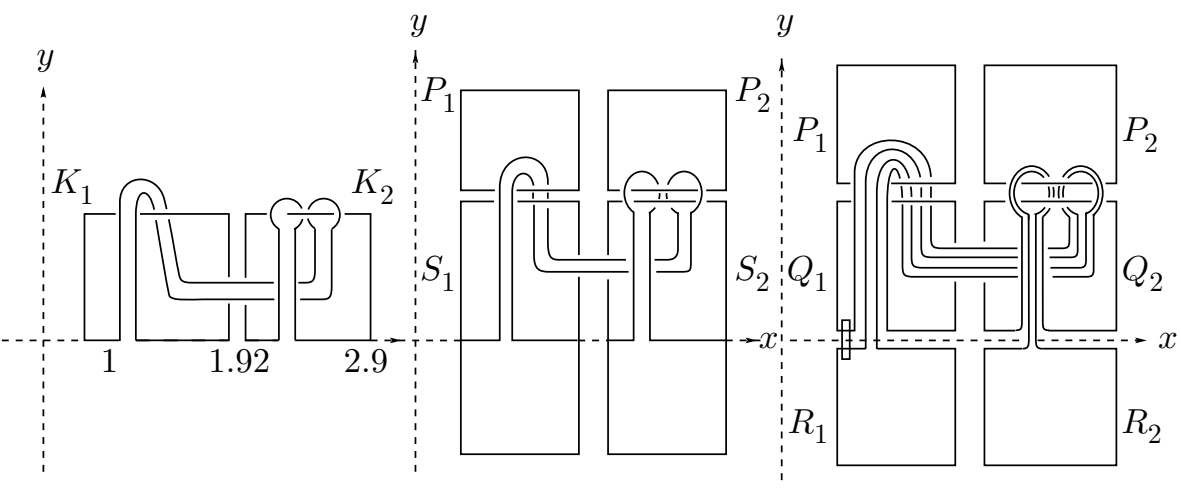

$(1)$

$(2)$

(3)

FiguRE II

out the band-fusion on $S_{i}$ by using the band $\widetilde{B_{i}}$ whose core is $l_{i}$. Then $S_{i}$ splits into two pieces. The one including the point $\{(i, 1,0)\}$ is called $Q_{i}$. The other is called $R_{i}$. Choose the band along $l_{i}$ so that $\operatorname{lk}\left(P_{i}, R_{i}\right)+\operatorname{lk}\left(Q_{i}, R_{i}\right)=0$. Here $P_{i}$ and $S_{i}$ are oriented counterclockwise, and $Q_{i}$ and $R_{i}$ are given orientations induced from $S_{i}$. (See Figure II(3).) Here, note that $\left(Q_{1}, \ldots, Q_{m}\right)$ defines the 1-link $L$. Thus we obtain a $3 m$-component 1 -link $L^{\prime}$ defined by $\left(P_{1}, \ldots, P_{m}, Q_{1}, \ldots, Q_{m}, R_{1}, \ldots, R_{m}\right)$ such that $L$ is a sublink of $L^{\prime}$.

Claim. There exists $f: S_{1}^{2} \amalg \ldots \amalg S_{m}^{2} \hookrightarrow \mathbb{R}^{3} \times \mathbb{R}$ such that $f$ is transverse to $\mathbb{R}^{3} \times\{0\}$ and $f\left(S_{1}^{2} \amalg \ldots \amalg S_{m}^{2}\right) \cap\left(\mathbb{R}^{3} \times\{0\}\right)$ in $\mathbb{R}^{3} \times\{0\}$ defines the 1-link $L^{\prime}$.

Proof. Construct an embedding $f: S_{1}^{2} \amalg \ldots \amalg S_{m}^{2} \hookrightarrow \mathbb{R}^{3} \times \mathbb{R}$ as follows. $f\left(S_{i}^{2}\right)$ in $\mathbb{R}^{3} \times \mathbb{R}$ has two minimum-discs, two saddle-bands and two maximum-discs. The minimumdiscs are $h_{i 1}^{0}=\{(x, y, z) \mid i \leqq x \leqq(i+0.9),-1 \leqq y \leqq 1, z=0\} \times\{-2\}$ in $\mathbb{R}^{3} \times\{-2\}$ and $h_{i 2}^{0}=\{(x, y, z) \mid i \leqq x \leqq(i+0.9), 1.05 \leqq y \leqq 2, z=0\} \times\{-2\}$ in $\mathbb{R}^{3} \times\{-2\}$. The saddle-bands are $\widetilde{B_{i}} \times\{-1\}$ in $\mathbb{R}^{3} \times\{-1\}$ and $\{(x, y, z) \mid i \leqq x \leqq(i+0.9)$, $1 \leqq y \leqq 1.05, z=0\} \times\{1\}$ in $\mathbb{R}^{3} \times\{1\}$. The maximum-discs are $h_{i 1}^{2}=\{(x, y, z) \mid$ $i \leqq x \leqq(i+0.9),-1 \leqq y \leqq-0.1, z=0\} \times\{2\}$ in $\mathbb{R}^{3} \times\{2\}$ and $h_{i 2}^{2}=\{(x, y, z)$ $i \leqq x \leqq(i+0.9), 0.1 \leqq y \leqq 2, z=0\} \times\{2\}$ in $\mathbb{R}^{3} \times\{2\}$, where we suppose $\partial \widetilde{B}_{i}$ 
$\cap S_{i}=\{(x, y, z) \mid x=i,(i+0.9),-0.1 \leqq y \leqq 0.1, z=0\} . f\left(S_{i}^{2}\right) \cap\left(\mathbb{R}^{3} \times\{t\}\right)$ $(-2<t<-1,-1<t<1,1<t<2)$ is an ordinary cross-section. Then $f\left(S_{1}^{2} \amalg \ldots \amalg S_{m}^{2}\right) \cap\left(\mathbb{R}^{3} \times\{0\}\right)$ in $\mathbb{R}^{3} \times\{0\}$ defines $L^{\prime}$.

Therefore the proof of Theorem 1.3 is complete.

Note. See $\S 2$ of [24] for the definitions of 'minimum-disc,' 'maximum-disc,' 'saddleband' 'ordinary cross-section,' etc.

Note. The 1-link $\left(Q_{1}, R_{1}\right)$ (or $\left.\left(P_{1}, Q_{1}\right)\right)$ is associated with a $\theta$-graph. The diagram of $\left(Q_{1}, R_{1}\right)$ (or $\left(P_{1}, Q_{1}\right)$ ) is what is used in the Appendix of [15]. Dr. Akira Yasuhara gave an alternative proof of [12], and wrote the Appendix of [15]. [11] is a generalization of [12] and [6].

Figure III (on the next page) illustrates $f\left(S^{2}\right)$ and $L^{\prime}$ in $\mathbb{R}^{3} \times \mathbb{R}$ in the case where $L$ is the trefoil knot. This method of drawing subsets of $\mathbb{R}^{3} \times \mathbb{R}$ is often used. See e.g. [1], [7], [9] and [24].

We next discuss ordinary sense slice 1-links in the case when we restrict the 2-knots of which the ordinary sense slice 1-links are cross-sections.

Theorem 3.2. For any $m$-component 1-link $L=\left(K_{1}, \ldots, K_{m}\right)$, there exist an embedding $g: S_{1}^{2} \amalg \ldots \amalg S_{m}^{2} \hookrightarrow \mathbb{R}^{3} \times \mathbb{R}$ and a 4 m-component 1-link $L^{\prime \prime}$ with the following properties.

(1) $g$ defines the trivial 2-link.

(2) $g$ is transverse to $\mathbb{R}^{3} \times\{0\}$, and $g\left(S_{1}^{2} \amalg \ldots \amalg S_{m}^{2}\right) \cap\left(\mathbb{R}^{3} \times\{0\}\right)$ in $\mathbb{R}^{3} \times\{0\}$ defines $L^{\prime \prime}$.

(3) $L$ is a sublink of $L^{\prime \prime}$.

(4) $K_{i} \subset f\left(S_{i}^{2}\right)(i=1, \ldots, m)$.

Ordinary sense slice $n$-knots $(n \geqq 1)$ which are cross-sections of the trivial $(n+1)$ knots are discussed in [13], [20], [21], and [22].

Proof of Theorem 3.2. In order to define $g$, take the following 2-discs $D_{i}^{2}$ in $\mathbb{R}^{3} \times \mathbb{R}$. Take $f$ in the proof of the above claim. $f\left(S_{i}^{2}\right) \cap \mathbb{R}^{3} \times(-1,2]$ are two components. Take the component of the two which includes $h_{i 1}^{2}$, say $E_{i}$.

$f\left(S_{i}^{2}\right) \cap \mathbb{R}^{3} \times[-2,1)$ are two components. Take the component of the two which includes $h_{i 1}^{0}$, say $E_{i}^{\prime}$. Then $\overline{E_{i} \cup E_{i}^{\prime}}$ is a submanifold in $\mathbb{R}^{3} \times \mathbb{R}$ diffeomorphic to the 2-disc. We call it $D_{i}^{2}$.

Take $D_{i}^{2} \times I$ in the tubular neighborhood of $D_{i}^{2}$ in $\mathbb{R}^{3} \times \mathbb{R}$. Take $g$ so that $g\left(S_{i}^{2}\right)$ coincides with $\partial\left(D_{i}^{2} \times I\right)$ and $g$ is transverse to $\mathbb{R}^{3} \times\{0\}$. Then $g\left(S_{1}^{2} \amalg \ldots \amalg S_{m}^{2}\right) \cap$ $\left(\mathbb{R}^{3} \times\{0\}\right)$ in $\mathbb{R}^{3} \times\{0\}$ is a $4 m$-component 1-link $L^{\prime \prime}$ and $L$ is a sublink of $L^{\prime \prime}$.

This completes the proof of Theorem 3.2.

Figure IV illustrates $g\left(S^{2}\right)$ and $L^{\prime \prime}$ in $\mathbb{R}^{3} \times \mathbb{R}$ in the case where $L$ is the trefoil knot.

Note 3.3. We can regard Figure IV as the example we mentioned in the note after Theorem 1.2 if we think of $L$ as a union of $K_{1}$ and $K_{2}$, and of $L^{\prime \prime}$ as a union of $K_{1}$, $K_{2}, K_{3}$ and $K_{4}$, where the $K_{i}$ are as in Figure IV.

Comparing Theorem 1.3 with Theorem 3.2, it is natural to raise the following problem. 

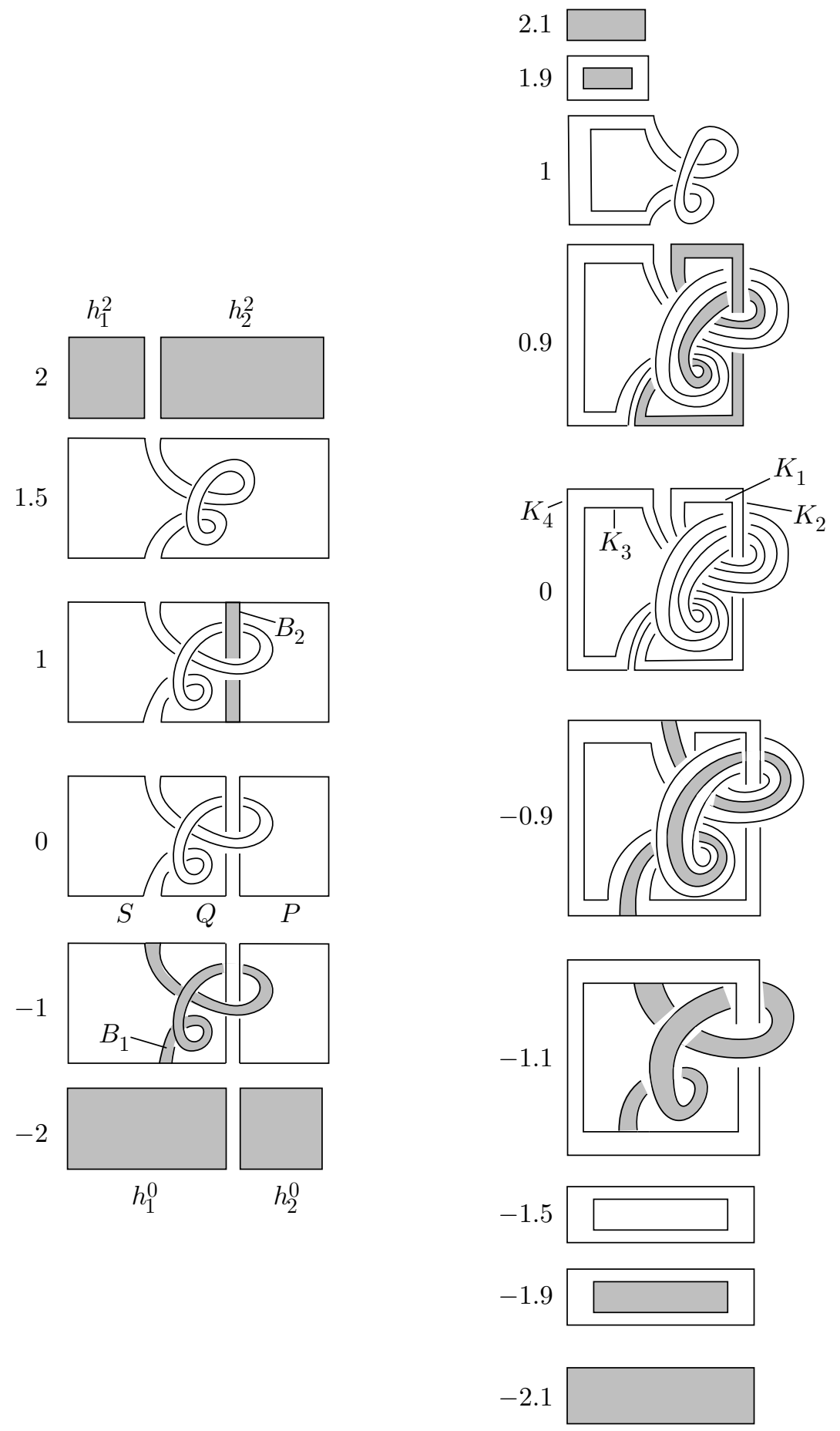

Figure III

Figure I 
Problem. Let $K$ be a non-slice knot. Does there exist a 3-component ordinary sense slice 1-link $L$ such that $K$ is a component of $L$ and $L$ is a cross-section of the trivial 2-knot?

Note 3.4. After Dr. S. Kamada saw this paper, he solved this problem and obtained a refined version of Theorems 1.3 and 3.2.

\section{ACKNOWLEDGEMENT}

When the author asked Prof. Akio Kawauchi whether Theorem 1.1 is new, Prof. Kawauchi answered affirmatively and encouraged the author to write it down. The author would like to thank Prof. Kawauchi for this advice. The author would also like to thank Prof. Shin'ichi Suzuki for valuable discussions, Dr. S. Kamada for the information incorporated in Note 3.4, and Dr. P. Akhmetiev for his interest in making our Theorem 1.1 stronger. He would also like to thank the referee for reading the manuscript patiently.

\section{REFERENCES}

[1] J. S. Carter and M. Saito, Knotted surfaces, braid movies, and beyond, Knots and quantum gravity, edited by J. C. Baez, Clarendon Press, Oxford (1994), 191-229. MR 95m:57036

[2] T. D. Cochran and K. Orr, Not all links are concordant to boundary links, Ann. of Math. 138 (1993), 519-554. MR 95c:57042

[3] R.H.Fox, Some problems in knot theory, Topology of 3-manifolds and related topics, Proc. 1961 Top. Inst. Georgia, Prentice-Hall, Englewood Cliffs, NJ, 1962, pp. 168-176. MR 25:3523

[4] P. Gilmer, Link cobordism in rational homology 3-spheres, J. Knot Theory Ramifications 2 (1993), 285-320. MR 94m:57012

[5] P. Gilmer and C. Livingston, The Casson-Gordon invariant and link concordance, Topology 31 (1992), 475-492. MR 93h:57037

[6] O. G. Harrold and S. Kinoshita, A theorem on $\theta$-curves and its application to a problem of T. B. Rushing, Bull. Acad. Polon. Sci. Sér. Sci. Math. 28 (1980), 631-634. MR 83h:57011

[7] S. Kamada, A characterization of groups of closed orientable surfaces in 4-space, Topology 33 (1994), 113-122. MR 95a:57002

[8] A. Kawauchi, On the Robertello invariants of proper links, Osaka J. Math. 21 (1984), 81-90. MR 85j:57004

[9] A. Kawauchi, T. Shibuya and S. Suzuki, Descriptions on surfaces in four-space, I. Normal forms, Math Sem. Notes Kobe Univ. 10 (1982), 75-125 II. Singularities and cross-sectional links Math sem. notes Kobe Univ. 11 (1983), 31-69. MR 84d:57017; MR 85j:57033

[10] M. Kervaire, Les noeudes de dimensions supérieures, Bull.Soc.Math.France 93 (1965), 225271. MR 32:6479

[11] S. Kinoshita, On $\theta_{n}$-curves in $\mathbb{R}^{3}$ and their constituent knots, In:Topology and Computer sciences, Kinokuniya, Tokyo (1987), 211-216. MR 92h:57010

[12] S. Kinoshita and H. Terasaka, On unions of knots, Osaka J. Math 9 (1957), 131-153. MR 20: 4846

[13] J. Levine, Doubly sliced knots and doubled disc knots, Michigan Math J. 30 (1983), 249-256. MR 85h: 57024

[14] J. Levine, Link invariants via the eta-invariant, Comment. Math. Helv. 69 (1994), 82-119. MR 95a:57009

[15] K. Miyazaki and A. Yasuhara, Generalized $\sharp$-unknotting operations, J. Math. Soc. Japan 49 (1997), 107-125. MR 97i:57007

[16] K. Murasugi, On a certain numerical invariant of link types, TransAMS 117 (1965), 387-422. MR 30:1506

[17] E. Ogasa, On the intersection of spheres in a sphere I, University of Tokyo preprint (1995).

[18] E. Ogasa, On the intersection of spheres in a sphere II:High dimensional case, University of Tokyo preprint (1995).

[19] R.A. Robertello, An invariant of knot cobordism, Comm. Pure. Appl. Math. 18 (1965), 543555. MR 32: 447 
[20] D. Ruberman, Doubly slice knots and the Casson-Gordon invariants, Trans. Amer. Math. Soc. 279 (1983), 569-588. MR 85e:57025

[21] D. Ruberman, The Casson-Gordon invariants in high-dimensional knot theory, Trans. Amer. Math. Soc. 306 (1988), 579-595. MR 89g:57031

[22] D. W. Sumners, Invertible knot cobordisms, Comment. Math. Helv. 46 (1971), 240-256. MR 44:7535

[23] S. Suzuki, Local knots of 2-spheres in 4-manifolds, Proc. Japan Acad. Sci. 45 (1969), 34-38. MR 40:2101

[24] S. Suzuki, Knotting problems of 2-spheres in the 4-sphere, Math. Sem. Notes Kobe Univ. 4 (1976), 241-371. MR 56:3848

[25] M. Yamamoto, Knots in spatial embeddings of the complete graph on four vertices, Topology Appl. 36 (1990), 291-298. MR 91m:57007

Department of Mathematical Sciences, University of Tokyo, Komaba, Tokyo 153, JAPAN

E-mail address: ogasa@ms.u-tokyo.ac.jp

E-mail address: ogasa@ms513red.ms.u-tokyo.ac.jp 